\title{
Measuring sustainability
}

\author{
Subhas K. Sikdar
}

Published online: 7 March 2012

(c) Springer-Verlag (outside the USA) 2012

In any seminar a sure amusement-inducing opening line is that there are as many definitions of sustainability as there are people in the audience. For scientists and engineers this is a problem of some magnitude. If we cannot know what sustainability is, how can we ascertain if we have attained it as a result of an intervention? If there is such a thing as sustainability science, as many have claimed, there must be more clarity about how we can determine a sustainable state. Many conceptual variations of the definition are not helpful. Part of the confusion emanates from the prevalent tendency of thinking in terms of global or regional sustainability, both of which will include measures that are beyond the reach of science or engineering alone. Policy attributes are also important in determining paths toward sustainability of these systems. Since the scope of this journal is primarily the practice of science and engineering, and includes policy discussions only as they are scientifically supportable, we can limit this discussion to the sustainability of products and processes.

We may never be able to describe a sustainable state that is static, but if we satisfy ourselves that a system under considerable is sustainable enough, it should be possible to describe the characteristics (i.e., indicators or metrics) of a dynamic system that remain the same through changes of the defining attributes (i.e., the system variables). In the last couple of decades the engineering researchers have thought of an easier approach by looking upon sustainability as a relative concept. Given that the traditional three dimensional considerations of environment, economy and society are

S. K. Sikdar $(\bowtie)$

National Risk Management Research Lab/USEPA, 26 W. M.L.

King Dr, Cincinnati, OH 45268, USA

e-mail: sikdar.subhas@epa.gov taken into account in an analysis, it is useful to determine if a newer product or process is "more sustainable" than the one being replaced. Likewise for a corporation or a place-based system, such as an ecosystem or an urban area, we would like to know if, over time, the system is moving towards or away from sustainability. It is thus not critical to know a sustainable state in an absolute sense; relative gain or loss of sustainability is sufficient for decision making. This concept is much like that of entropy, for which entropy change is the quantity that is useful, not its absolute value. Assuming that we have made an improvement in a system as revealed by better values of its sustainability indicators, we will make progress in sustainability sense without really knowing how much more progress can still be made. This is not necessarily a bad outcome. However, there is one problem on which improvement is badly needed. We need a scientifically sound understanding of the indicators or metrics we use for sustainability analyses.

Certain suggestions can be made towards the goal of attempting sustainability analyses.

- First state clearly what system is being considered for sustainability analysis. This statement would have to show the system boundary.

- Second, consider all possible indicators or metrics that would describe the system for a sustainability analysis. These indicators must be quantifiable.

- Third, prioritize the indicators or metrics so that only the necessary and sufficient number of indicators is included. This implies that negligible error in decision is encountered by excluding some indicators.

- Fourth, conduct an analysis that provides quantified data on the environmental, economic, and societal impacts (benefits and costs) for the system alternatives in question. 
- Lastly, use a method or an algorithm that allows decision making on the alternatives or on the temporal states of a system.
Much research needs to be conducted on all these steps so that claims of sustainable practice can be accepted as credible. 